\title{
A rationalised virological electron microscope specimen testing policy
}

\author{
A Curry, A Bryden, P Morgan-Capner, A Fox, M Guiver, L Martin, K Mutton, P Wright, \\ P Mannion, A Westwell, J Cheesbrough, I Ashton, A Blackley \\ (PHLS North West Viral Gastroenteritis and Electron Microscopy Subcommittee)
}

A Blackley

Public Health

Laboratory, Royal

Preston Hospital,

PO Box 202, Sharoe

Green lane, Preston

PR2 9HG, UK

A Bryden

L Martin

P Wright

J Cheesbrough

Public Health

Laboratory, Fazakerley

Hospital, Lower lane,

Liverpool L9 7AL, UK

K Mutton

I Ashton

Public Health

Laboratory, Countess of Chester Health

Park, Liverpool Road, Chester CH2 1UL, UK P Mannion

PHLS North West Headquarters, Vicarage Lane, Fulwood, Preston PR2 8DY, UK

P Morgan-Capner A Westwell

Correspondence to: Dr Curry.

Accepted for publication 28 January 1999

\begin{abstract}
Public Health
Laboratory,

Withington Hospital, Manchester M20 2LR, UK

A Curry

A Fox

M Guiver

Abstract

The aim of this project was to produce guidance for a rationalised virological electron microscopy specimen testing policy for PHLS North West, to facilitate centralisation of a groupwide diagnostic electron microscopy service on a single site. Careful specimen selection to limit numbers and the groupwide use of commercially available enzyme immunoassays has allowed PHLS North West to reduce the number of specimens prepared for electron microscopy. The rationalised virological electron microscopy specimen testing policy has enabled a diagnostic electron microscopy service to be provided from a single site with a manageable workload. Implementation of this specimen testing policy by PHLS North West has been successful and may be applicable to other laboratories (or groups of laboratories) to maximise the use of expensive electron microscopy facilities.

(F Clin Pathol 1999;52:471-474)
\end{abstract}

Keywords: electron microscopy; virology; rationalised specimen testing policy

Rationalisation of expensive diagnostic services, such as electron microscopy, is an inevitable consequence of the tight financial pressures that encompass all aspects of delivering a diagnostic pathology service. Electron microscopy has high capital costs (particularly if equipment is bought new), requires highly skilled and therefore expensive staffing, and can only deal with a limited number of specimens, as each one is examined individually. Depending on the number of specimens examined and the grading of the staff involved, the cost of preparation and examination of negatively stained samples for electron microscopy can be up to $£ 30$ each. Within the Public Health Laboratory Service (PHLS), grouping of laboratories has allowed centralisation of some diagnostic services to maximise use of expensive equipment and thus provide a more cost-effective service. Originally the four laboratories (Chester, Liverpool, Manchester, and Preston) comprising PHLS North West all provided a local electron microscopy service. Centralisation of these services into a single electron microscopy unit based at the Manchester laboratory provided the stimulus to look at a rationalised specimen testing policy. Three main areas of diagnostic virology had been provided previously by electron microscopy in larger public health laboratories, namely, the investigation of outbreaks of non-bacterial gastroenteritis, the investigation of sporadic cases of infantile gastroenteritis, and the rapid examination of skin lesions. A groupwide testing policy has been implemented to reduce the workload of the electron microscopy unit by testing only those specimens necessary for accuracy of diagnosis and patient management, and provide focused surveillance data. Careful selection and limitation of numbers of specimens and the use of commercially available enzyme immunoassays (EIA) has allowed the PHLS North West Group to undertake a groupwide diagnostic service on a single site while still providing a high quality service.

\section{Methods}

The PHLS North West Group of laboratories serves a large catchment area in the north west of England. The electron microscopy specimen testing protocol has been circulated to all consultant microbiologists, consultant virologists, consultants in communicable disease control (CCDCs), and environmental health departments within this area.

Successful application of the electron microscopy specimen testing protocol is dependent on other facets of the PHLS North West group's infrastructure, particularly the group transport system, the common group IT computer system (Telepath), and interlaboratory communication via the IT "mailbox" facility or by telephone.

In addition to careful specimen selection it is axiomatic that the methods used to prepare specimens for electron microscopy must optimise the chances of detecting and identifying virus particles, if present, by partial purification and concentration. Recommended methods for stool preparation are double spin centrifugation $^{1}$ or ammonium sulphate precipitation. ${ }^{2}$ The first method is used routinely, but the second method is used if the ultracentrifuge fails or is being serviced.

PHLS NORTH WEST ELECTRON MICROSCOPY SPECIMEN TESTING POLICY

Selection of the most appropriate samples for testing has to be based on the clinical details provided. These should include age of patient, symptoms, and the date of onset, and, if associated with an outbreak, be accompanied by the details of location of the outbreak, the numbers involved, and the possible source. Specimens submitted without this information may not be tested until details are supplied, although those with insufficient information will be retained 
for at least a week, so that adequate details may be obtained retrospectively.

\section{OUTBREAK INVESTIGATION}

The investigation of outbreaks is a PHLS priority and the rationalised electron microscopy service had to meet this requirement.

Each laboratory in the group has appointed a coordinator who should be aware of possible local outbreaks. This individual coordinates the laboratory investigations of the outbreak specimens and is responsible for correlating and disseminating results. All inquiries about an outbreak are directed to the coordinator. If the outbreak is thought to be of viral origin, then the outbreak coordinator sends an appropriate batch of specimens to the centralised electron microscopy unit for testing.

The following features are suggestive of an outbreak of viral gastroenteritis ${ }^{3}$ :

- 15 to 48 hour incubation period

- vomiting in $>50 \%$ of cases

- duration of illness 12-60 hours

- high secondary attack rate

- stool cultures negative for bacterial pathogens, if results available.

OUTBREAK SPECIMEN SELECTION AND TESTING

Specimens for electron microscopy examination should be:

- unformed faecal specimens (not formed stools or vomitus)

- collected from symptomatic individuals

- taken within three days of onset

An initial batch of up to six specimens is examined by electron microscopy. If two or more of this initial batch are positive, then subsequent samples are reported as "not testedpart of a positive outbreak".

A further batch of four specimens is examined if none of the initial batch is found positive, or only a single sample is positive (possible coincidental result), and the outbreak remains bacteriologically negative.

This means that results on all specimens submitted from an outbreak should not be expected.

In hospital outbreaks, new cases on a different ward within the same hospital are regarded as a potentially new outbreak. In such cases a small number of samples (a maximum of four) from those "new" wards are tested to determine if the same or a different agent is involved. Similarly, if new cases occur on any previously affected ward more than a week after the last case of the initial outbreak, a small number of samples (maximum of four) is tested to determine if the same or a different agent is involved.

In outbreaks involving homes for the elderly, electron microscopic examination of the submitted specimens will often show viruses other than small round structured viruses (SRSVs). Viruses such as rotaviruses which are normally associated with infantile gastroenteritis can be found in the elderly because of degraded immunity. If rotaviruses or adenoviruses are identified, then subsequent specimens can be tested by the appropriate enzyme linked immunoassay (EIA), if necessary.
Results are usually available within two working days of receipt of the specimens by the electron microscopy unit. Positive results are telephoned, provided a contact name and number have been provided. Negative results are reported either electronically or as printed reports.

SPORADIC CASES OF INFANTILE GASTROENTERITIS Rotavirus and adenovirus EIAs are undertaken within all group laboratories. Electron microscopic investigation of EIA negative samples is undertaken on local samples submitted to Manchester Public Health Laboratory (demographic details available) and from a children's hospital in Liverpool (contractually specified).

\section{Specimen selection and testing}

From general practices, specimens are tested from children five years of age or under, with diarrhoea and/or vomiting. All children admitted to hospital with symptoms of gastroenteritis, and appropriate immunocompromised patients, will be tested irrespective of age. In exceptional circumstances, specimens from other patients may be examined after discussion with a consultant microbiologist.

Specimens sent for examination should be:

- unformed faecal specimens (not formed stools or vomitus)

- collected from symptomatic individuals

- taken within three days of onset

Specimens fulfilling the above criteria are tested initially using EIA for rotavirus and adenovirus. If any specimen is positive by EIA for rotavirus or adenovirus (or both), the result is reported and no further investigation carried out. If, however, the rotavirus and adenovirus EIA tests are negative and the child is three years of age or under, electron microscopy is undertaken.

Results are usually available within three working days of receipt and are reported electronically or as printed reports.

SKIN LESIONS

Urgent skin lesion specimens from immunocompromised patients are given priority and following previous notification are examined as soon as they are received in the electron microscopy unit (working hours only); results are reported by telephone. Non-urgent skin lesions are examined with other routine electron microscopy specimens and results reported either electronically or as printed reports.

In addition, specimens from patients with possible poxvirus infections, where electron microscopy is the only readily available diagnostic method, are also tested as soon as possible.

Dried vesicle fluid on a glass microscope slide, vesicle fluid in a capillary tube, or a piece of the skin lesion in a dry sterile bijoux bottle are accepted for examination by the electron microscopy unit. In addition, a lesion swab, in virus transport medium, should be sent for virus isolation in cell culture. 
Results

All four laboratories comprising PHLS North West had originally provided a local electron microscopy service, examining a total of about 8500 specimens a year (Chester 1000; Liverpool 3000; Manchester 3500; Preston 1000). Following grouping, rationalisation of the electron microscopy services on one site (Manchester), and implementation of the rationalised electron microscopy specimen testing policy, the electron microscopy workload at Manchester was stabilised at about 3500-4000 specimens a year (not including the specimens tested by EIA).

\section{Discussion}

The electron microscopy units at Preston PHL and Chester PHL were to be decommissioned because of a change in PHLS policy which favoured core funding of a smaller number of strategically located electron microscopy facilities. With grouping of laboratories within the PHLS, further rationalisation and centralisation was possible.

The specimen testing rules outlined here have allowed the PHLS North West group to maintain a manageable electron microscopy workload and provide a high quality service. However, a limiting element in this process, particularly in outbreak investigations, is the transport of specimens from source to laboratory. Electron microscopy can produce relatively rapid results from some specimens, at least, if they arrive early enough. Transport from outbreak source to receiving laboratory, followed by further transport to the testing laboratory, inevitably induces delays, which are frustrating for those responsible for managing an outbreak. Lack of local laboratory electron microscopy provision may be a source of annoyance, but it must be borne in mind that the centralised electron microscopy service is still going to be at least as quick as bacteriological testing.

At the testing laboratory, an essential part of the algorithm is specimen reception, where accurate entry of appropriate data on to the group computer system allows appropriate specimen selection and worksheet generation of specimens to be tested. The rule base outlined above facilitates the smooth flow of work within the laboratory.

The group electron microscopy testing policy has been sent to all consultant microbiologists, consultant virologists, CCDCs, and environmental health departments, and our changed provision has been accepted without adverse comment. Over time, it is hoped that the most appropriate specimens and the required clinical details will be submitted directly to the receiving laboratory and that this will produce a more efficient booking in, selection, and processing system.

SRSVs have been identified in vomitus ${ }^{4}$ which is comparatively easy to collect in outbreaks in institutions, such as hospitals or homes for elderly persons. However, from experience (unpublished results), stool specimens from affected individuals are significantly more productive. Therefore, the North West group electron microscopy policy recommends examination of faecal samples rather than vomitus. Likewise, loose or unformed stools are preferred to fully formed samples, as 20 years of experience have shown (unpublished results) that these are more productive. However, this aspect of the policy may be controversial, as other centres ${ }^{5}$ suggest that fully formed stools should not be eliminated from testing rules. Because of the workload on the group electron microscopy unit, only specimens considered to have the greatest likelihood of being positive are examined. Thus samples from asymptomatic individuals involved in outbreaks, who may be excreting virus, would not be included, as the probability of finding virus in such samples is limited.

The large numbers of sporadic cases of infantile gastroenteritis, particularly during the winter months, could on their own overload the group electron microscopy facility. The introduction of the rotavirus and adenovirus EIA testing on specimens from children aged five years and under has allowed the North West group to identify the majority of infections with these agents, the most common causes of infantile viral gastroenteritis. ${ }^{6}$ These EIAs, by their very nature, allow all the group laboratories to handle relatively large numbers of specimens and to screen out many positives. An additional benefit is that EIAs are relatively easy to interpret and are comparatively cheap, especially compared with electron microscopy. The results of the EIA tests are available at the same time as, and are reported simultaneously with, bacteriological results.

The selection of the five years and under age limit for EIA testing is arguable, but analysis of positive results for enteric viruses (except SRSVs which, from outbreak data analysis, can infect any age group) has shown a peak incidence at two years of age; thus it was considered that five years and under was a reasonable compromise, given that only a few slightly older children are likely to be positive.

The testing by electron microscopy of ELISA negative samples from children three years old and under is again arguable, but it has been shown that the majority of infantile viral gastroenteritis infections occur before the age of two years. ${ }^{6}$ Most rotavirus and adenovirus infections will be detectable by EIA alone; other viral causes of gastroenteritis - such as astroviruses, caliciviruses, and SRSVs-will, although relatively infrequent, be detected by electron microscopy. By limiting electron microscopic examination of cases of infantile diarrhoea in the catchment area of the centralised electron microscopy unit, the results can be correlated with local demographic details. All such data on viral gastroenteritis are transmitted to the PHLS Communicable Disease Surveillance Centre (CDSC) so that any changes in the pattern of enteric virus infection can be monitored.

Under ideal conditions only about $25 \%$ of specimens from outbreaks may be positive for SRSVs by electron microscopy. In addition, with the use of EIAs for rotavirus and adenovirus, the majority of positive specimens will be 
screened out before electron microscopy testing is undertaken. This means that finding virus positive specimens by electron microscopy may become relatively infrequent, particularly during the summer months, possibly affecting the confidence of the microscopist. To maintain interest and competence, it is important that known positive samples for all relevant viruses should be reintroduced into the electron microscopy system on a regular basis. This should form part of the laboratory quality assurance procedures and be organised by the quality assurance (QA) manager from stored specimens.

The polymerase chain reaction (PCR) can be used to detect SRSVs in stool samples ${ }^{7}$ and a limited SRSV PCR diagnostic service is available within the PHLS. Although electron microscopy remains the "front line" diagnostic technique, PCR can be applied to epidemiological investigations. In several centres, developments are underway to produce an EIA suitable for the detection of SRSVs. Using molecular methods, recombinant capsids of SRSVs have been produced and antibodies produced from these. These reagents will ulti- mately provide an EIA able to detect all circulating SRSV genotypes. Once available, such an EIA would have significant advantages over electron microscopy in SRSV detection and would necessitate further re-evaluation of electron microscopy in diagnostic virology. Because electron microscopy is a "catch all" diagnostic method, a small number of electron microscopy units would have to be retained so that any potential emergence of a new antigenic type or re-emergence of types not covered by available EIAs could still be detected.

1 Riordan T, Craske J, Roberts JL, et al. Food borne infection by a Norwalk-like virus (small round structured virus). $\mathcal{F}$ Clin Pathol 1984;37:817-20.

2 Caul EO, Ashley CR, Eggleston S. An improved method for the routine identification of faecal viruses using ammonium sulphate precipitation. FEMS Microbiol Lett 1978;4:1-7.

3 Caul EO, Sellwood NJ, Brown DW, et al. Outbreaks of gasCaul EO, Sellwood NJ, Brown DW, et al. Outbreaks of gas-
troenteritis associated with SRSVs. PHLS Microbiol Digest 1993;10:2-8.

4 Greenberg BB, Wyatt RG, Kapikian AZ. Norwalk virus in vomitus. Lancet 1979;i:55.

5 McCaughey C, O'Neill HJ, Wyatt DE, et al. Effect of faecal consistency on virological diagnosis. F Infect 1998;36:1458.

6 Ellis ME, Watson B, Mandal BK, et al. Micro-organisms in gastroenteritis. Arch Dis Child 1984;59:848-55.

7 Lambden PR, Caul EO, Ashley CR, et al. Sequence and genome organisation of a human small round structured (Norwalk-like) virus. Science 1993;259:516-19. 